\title{
Research of Real-time Face recognition method on mobile intelligent device
}

\author{
Wu LV ${ }^{1, a}$, Tian-huang $\mathrm{CHEN}^{2, \mathrm{~b}}$ \\ School of computer science and technology, Wuhan University of Technology, Wuhan, Hubei, \\ 430063, China \\ a446951851@qq.com, bthchen57@126.com
}

Keywords: Face recognition; Template Matching; Facial Features Localization; Android

\begin{abstract}
In order to solve the problem that traditional face recognition scheme was not efficiently suitable for the smart mobile terminal. This paper proposes a Real-time Face Recognition Method based on geometric features and improved template matching. The method is divided into two steps from general to detail for recognition, the first step is to detect the similarity of key geometric features of face, the second step is to match the detail features of the eyes, nose, mouth by using a specific feature matching template. The experimental results show that the algorithm has good performance in speed and recognition rate, and it has important value of practical application.
\end{abstract}

\section{Introduction}

The development of mobile Internet makes mobile phones have more and more functions. They are used not only to pay, but also to manage many private information of users, so the security of mobile applications attract more and more attention. At present, biometric identification has become a more reliable basis. Among many biometric technologies, face recognition has the advantages of non-contact, convenient and direct, friendly, reliable etc, so using face recognition technology as the security identification method fort the intelligent mobile terminal application is a natural idea. However, due to the limited computing power and storage space of the mobile intelligent terminal system, the traditional face recognition algorithm is not effective when it is applied to the intelligent terminal directly ${ }^{[1]}$.

In China, there are few researches on the face recognition on mobile intelligent terminal is relatively small, there are some algorithms that directly transplanted from PC side, and the running efficiency is low. Literature [2] is based on the Symbian client, using the LBP algorithm to do face recognition, and put forward a complete set of solutions, The problem is that the platform is older. Wang Er wei, etc. transplanted Adaboost face detection algorithm to the Android platform, and use PCA algorithm to extract features to do the face recognition ${ }^{[3]}$, But before the recognition, it needs a large number of samples to train, which takes a long time, and it is not convenient to use. Lv Wei, etc. use skin color features to achieve the face detection on Android platform, and then do face recognition based on LBP algorithm ${ }^{[4]}$, The recognition speed can meet the real-time requirement, but the recognition rate is only $83.5 \%$. Wang Hong bin proposed a face detection system on Android which based on the computer vision library OpenCV, and introduced the recognition method and the algorithm transplant process in detail. ${ }^{[5]}$. Yang Wei guo has made the analysis to the limit difficult problem about how mobile phone platform to solve the large amount of computation and real-time operation in the article " rapid smiling face identification method for the mobile phone platform", the process of identification and recognition algorithms has been described ${ }^{[6]}$. In the literature [7], the face detection and authentication system based on mobile phone platform is designed, but it has not been tested on the mobile platform. From the discussion above, we can see that the domestic face recognition and authentication research on mobile phone platform is still in the initial stage, has not yet entered the practical field, it worths studying further

Based on this background, this paper proposes a real-time face recognition method based on geometric features and improved template matching. To increase the speed of the extraction of 
geometric features, geometric feature extraction is done in the thumbnails from original high-definition map which is reduced the size in accordance with certain proportion, then to get the corresponding points in original high-definition map based on the proportion of scaling and rotation after determined the geometric features of key points, details area of eyes, nose and mouth are cut according to these key points. Combining with the characteristics of contour lines of eyes, nose and mouth when doing detail area matching, ignoring minor feature information, so we need not compute each pixel in the template, it can save half of the calculation time. The amount of calculation of this method is much smaller than that of the traditional face recognition algorithm. It is developed and tested on Android platform, the speed of recognition achieve real-time requirements and the recognition rate is well.

\section{Method introduction}

The face image to be verified is captured from the mobile phone camera. Then extract the pupil, the nostrils and mouth six geometric features of facial key points, calculate the Euclidean distance between feature points to obtain a set of feature vectors. And this set is used to calculate the similarity with the pre-stored face geometric feature vectors of the mobile phone owner. The match will if it can't reach the threshold. If it can reach, then it can come to the second step: cut areas of eyes and nose and mouth according to the geometric feature points in the face image normalized, then use special template of eyes and nose and mouth calculated by the pre-stored face image to match the cutting area image, finally calculate the comprehensive similarity according to the similarity of eyes and nose and mouth by different weight, the match is successful if it can reach the preset threshold value, otherwise failure.

According to the characteristics that the mobile phone face recognition image is clear and the attitude changes little, the method of this paper achieves real-time verification by calculating and storing more standard face image feature data to reduce feature extraction time of being verified and improve template matching speed, and ensure a good recognition effect. Face detection and eye positioning is carried out in the thumbnails which comes from the original image but is reduced in a certain proportion. The corresponding points in the original image can be found according to the scaling ratio and the detection results, After the face detection and eye positioning, do normalized operation on the thumbnail, being followed by the nostrils and mouth corner location. Doing rotate and zoom operation on the original image to make the pupillary distance of the standard face image and the HD original image be the same. This ensures that the template can keep consistent with the target size when matching.

\section{Geometric feature extraction}

Classifier is the modeling technique of target discrimination which focuses on the research in field of pattern recognition. In recent years, researchers have applied the idea and method of classifier to the problem of facial feature point location. The classifier can give a certain discrete or continuous output based on the given input, so it can be used to determine the search direction of the feature points directly. On the one hand, the way of classifier technology applied to the facial feature location can be directly into the model to search the feature points, or directly into the model like regression device to predict the location of features; on the other hand, we can optimize the energy function extracted from features by using mature learning and optimization methods of classifier ${ }^{[8]}$.

Firstly doing face detection based on cascade classifier. The better trained classifier will have a faster detection speed and higher recognition rate. Before the pinpoint of the pupil, the nose and the mouth, the classifier is used to locate the approximate cross position. Designate approximate detection area according to the priori knowledge of "three court five eyes" facial features, and three court five eyes ratio is shown in figure 1. 


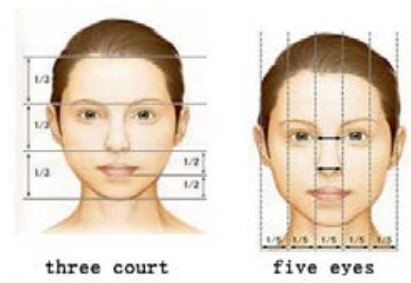

Figure 1 schematic diagram of three court five eyes

The positioning sequence of the feature points is the pupil, followed by the nose and the mouth. The detection area of the nose needs to refer to the position of the pupil, and the position of the mouth needs to refer to the position of the pupil and the nose. Experiments show that the positioning of the eye classifier can be located on the eyeball, but can not locate the position of the pupil. The solution is to do eye template matching in the neighborhood of $10 * 10$ centered at the initial location point to determine the exact location of the pupil, the eye template is shown in figure 2.

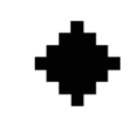

Figure 2 Eye search template

After determining the position of the pupil, you can calculate the angle between the eyes and the horizontal direction, and rotate, then, cut and scale the image according to the pupillary distance, finally get the normalized face image with the pupillary distance to be D. The nostrils and mouth corners can be positioned in the normalized image. The geometric normalization process is shown in figure 3 .

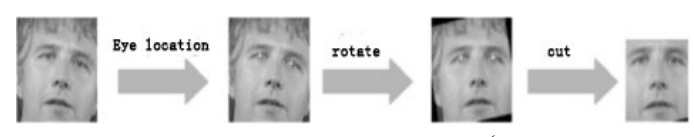

Figure 3 Geometric normalization process (from ORL face database)

The gray value of the nostril is lower than that of the areas near the nostril. According to this characteristic, we can locate the nostrils in the detected region by classifier, and the corners of the mouth position can use the most prominent horizontal line when the mouth is closed. The line can be obtained through the edge detection, and the two ends of the line are two corners of the mouth. Feature extraction results are shown in figure 4.

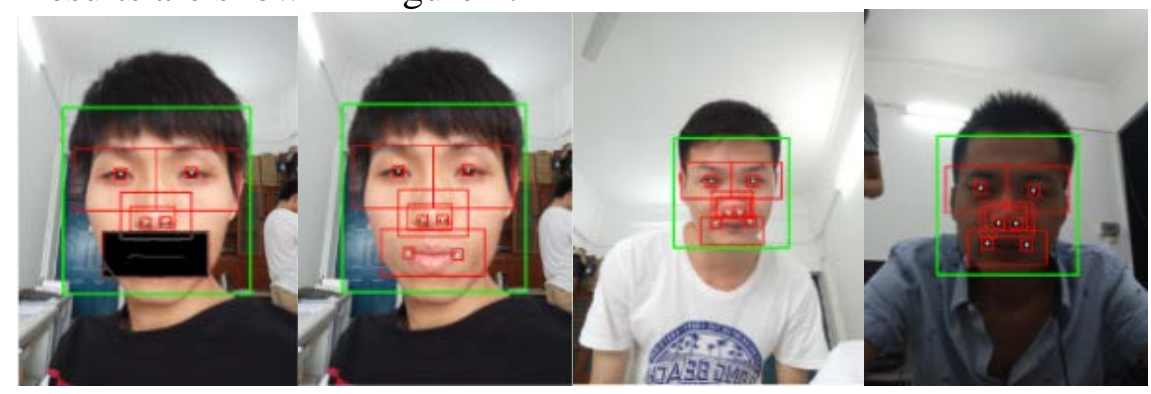

Figure 4 Samples of Geometric feature extraction

The relative Euclidean distance of the feature points on the normalized image is calculated. Feature vector includes the distance between the pupil and the nostril, the distance between the pupil and the mouth (the left and right eyes, the left and right corner of the mouth, a total of four distance data), the vertical distance between the pupil and the nostril, the vertical distance between the pupil and the corner of the mouth, the distance between the two corners of the mouth, the distance between the two nostrils. The feature vector can be obtained as each of the distance above divided by the papillary distance, then the feature vector has nothing with the size of the picture. Finally the feature vector has 8 dimensions.

The above geometric feature extraction process is used for the face image to be verified. However, the standard face image stored in the mobile phone needs to not only position the six feature points,. but also locate the four corners of eyes and detect the edge line of upper and lower eyelids, nose and lips in order to cut out the standard template to use. The feature information of the standard face image is computed only once, so it is acceptable to detect the feature points for some time. 


\section{Improved template matching}

Image matching technology is to use the known template to match the image to be recognized by an algorithm to calculate whether the image has the information and coordinates of the template. The commonly used similarity measure is variance, correlation coefficient, normalized variance, normalized correlation, and normalized correlation coefficient. The calculation of variance is less, but the matching effect is poor. Weighing the amount of computation and the matching effect, in this paper, we use the correlation coefficient method. Calculate as follows:

$$
\begin{aligned}
& R(x, y)=\sum_{x^{\prime} y^{\prime}}\left(T^{\prime}\left(x^{\prime}, y^{\prime}\right) \times I\left(x+x^{\prime}, y+y^{\prime}\right)\right) \\
& T^{\prime}\left(x^{\prime}, y^{\prime}\right)=T\left(x^{\prime}, y^{\prime}\right)-1 /(w+h) \times \sum_{x^{\prime \prime} y^{\prime \prime}}\left(T\left(x^{\prime \prime}, y^{\prime \prime}\right)\right.
\end{aligned}
$$

$\mathrm{S}$ is the image to be matched, $\mathrm{I}$ is the sub graph matrix to be matched with the template in $\mathrm{S}$. $\mathrm{T}$ is the template matrix, $\mathrm{R}$ is the result matrix. When the template and the sub graph are exactly the same, the correlation coefficient $\mathrm{R}(\mathrm{x}, \mathrm{y})=1$, and the worst match is -1 . The maximum value of $\mathrm{R}$ is found after the full search is completed in $\mathrm{S}$, and the corresponding sub graph is the best matching target.

In the calculation of standard template matching, each pixel point of the entire template region is calculated to get the matching similarity. Some of the pixel information in the template matching is very important, but some of the pixel information on the matching results not only has no positive effect, but also even lower the similarity. The difference is mainly reflected by each person's eyelids, eye haustra and orbital. So we can only calculate the contour line area around the eye when doing eye template matching, use the main features of the eyes to distinguish the similarity of two eyes. Similarly, the nose and mouth also have a characteristic contour feature. Based on this consideration, in order to reduce the amount of calculation, do special template matching according to the features of the eyes, nose and mouth. Detect the unique contour lines of the eyes and nose and mouth in the template, then calculate the pixels around the contour line area which takes the contour line as the center and 5 pixel width to match. Contour features are shown in figure 5.

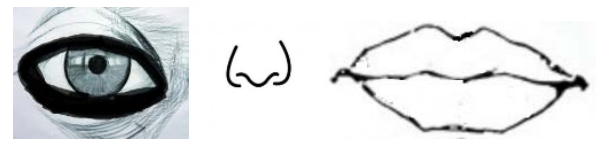

Figure 5 Contour line of eyes, nose and mouth

In order to make sure the size of the target and the template is consistent, the standard template and the image to be matched must be guaranteed to be cut with the same pupillary distance, otherwise it is impossible to match successfully. The calculation of the template matching is related to the size of the template itself, so we need to select the appropriate pupillary distance to increase the matching speed. Determine the location of the feature points in the standard template by using more elaborate algorithms, and cut out template image as small as possible, at the same time, ensure enough feature information. When we cut the image to be matched, the size of it can be appropriately enlarged, so that the space of the target can be in the cutting area as much as possible.

\section{Similarity measure}

The two geometric feature vectors are $\mathrm{P}$ and $\mathrm{Q}$ respectively. The geometric feature similarity formula is as follows:

$$
\left.\mathrm{C}=1-\sqrt{\sum_{i=1}^{8} P_{i}-Q_{i}^{2}} / \sum_{i=1}^{8} P_{i}\right) \times 100 \%
$$

$\mathrm{P}$ is the pre-stored feature vector of the standard face image, $\mathrm{Q}$ is the feature vector of the face image to be verified through the real-time calculation. According to the result of the test, the best similarity threshold of geometric feature is about $93 \%$ as the best recognition rate. The similarity of geometric features is shown in figure 6. 


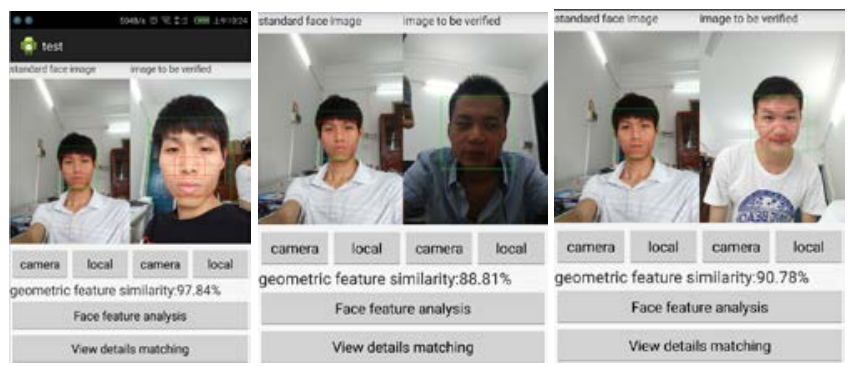

Figure 6 Comparison of geometric similarity

Weighing the amount of computation and the matching effect, we use the correlation coefficient method., matching results are shown in figure 7.

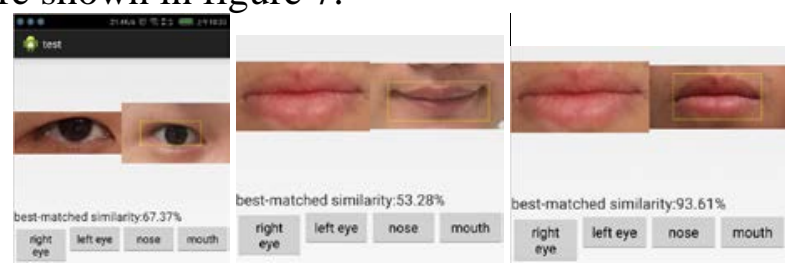

Figure 7 Details match results

To find the best matching position in the result matrix and get the matching similarity results. Calculate the comprehensive similarity according to the importance of eyes, nose and mouth. According to the change of the eye, nose mouth caused by light and angle, setting weight is shown in table 1.

Table 1 The weight proportion of the face region

\begin{tabular}{|c|c|}
\hline region & weight \\
\hline left eye & 0.2 \\
\hline right eye & 0.2 \\
\hline nose & 0.25 \\
\hline mouth & 0.35 \\
\hline
\end{tabular}

\section{Experimental results and analysis}

System tests based on Android: to develop the C++ dynamic link library (.So) by using NDK programming and JNI has higher runtime efficiency than that of the native Android which is developed by java. During the test process the ZTE Nubia Z9mini mobile phone is used. This kind of mobile phone is equipped with CPU of Qualcomm snapdragon 615, 2 GB memory, 8 million pixel camera (front), and the system version is Android 5.0.

Because of the influence factors such as the angle of view, pose, facial expression and so on, the ORL face database is not suitable to be used as the test image for the mobile phone. So in this paper, the human face images to be tested is taken by the mobile phone camera or come from the network. The image which is selected because of factors such as normal illumination, opened eyes and closed mouth, natural facial expression and the facial rotation is small. Obtain many groups of similar face images to test from the network, because the similar face image has an important reference for the results of the algorithm test.

The test is divided into two groups of 300 images. One group is one to one that it is for two different face images of the same person with a certain attitude angle and the change of illumination. The other group is one to many that it is to find the same person face image with the template in a number of similar face photos. Literature [10] and literature [3-4] experimental test image are also from mobile phone camera, the recognition rate improves with the increaseing dimension of the feature vector in literature [3], and the overall performance of the ORL database is better than the self-built database. It is not the face recognition algorithm only used for the mobile phone camera.the algorithm test results are shown in table 2. 
Table 2 recognition results of statistics

\begin{tabular}{|l|c|c|c|c|}
\hline & $\begin{array}{c}\text { recogni } \\
\text { tion rate }\end{array}$ & $\begin{array}{c}\text { Time } \\
\text { consuming } \\
(\mathrm{ms})\end{array}$ & $\begin{array}{c}\text { Miss } \\
\text {-recognit } \\
\text { ion rate }\end{array}$ & $\begin{array}{c}\text { false } \\
\text { recognition } \\
\text { rate }\end{array}$ \\
\hline Method in literature [3] & $60 \%-85$ & 133.541 & NULL & NULL \\
$\%$ & $83.5 \%$ & 93.3 & NULL & NULL \\
\hline Method in literature [4] & $85.5 \%$ & 123 & NULL & NULL \\
\hline Method in literature [14] & $93.6 \%$ & 217.5 & $1.6 \%$ & $4.8 \%$ \\
\hline Method in this paper &
\end{tabular}

It can be seen that the recognition rate of the method in this paper is higher, and the recognition speed can also be used in real-time application.

\section{Conclusions}

This paper proposes a real-time face recognition method based on geometric features and improved template matching: firstly locate the approximate position of geometric features by cascade classifier, and then locate precise six feature points localization of the pupil, the nostrils and mouth through the appropriate algorithm. If the geometric feature similarity threshold achieves the set threshold, it can enter the second step to do detail feature matching of eyes, nose and mouth, and the matching area is cut by the geometry feature point, the template is form the pre-stored standard face images through accurate calculation. Get the final recognition results by comprehensive details data. Experimental results show that this method is effective and has practical application value. However, the recognition images are static, so it can also be recognized successfully by using a picture of the mobile phone owner, so, it is need to have a deeper study in the case of fraud.

\section{Reference}

[1] Haidong Zhou. Research and implementation of intelligent terminal lightweight face recognition system [D]. XiDian university, 2014.

[2] CHEN Y N,CHEN X G.A novel identify recognition system based on mobile phone and face recognition[J]. Computer Application Software,2011, 28(3): 77-79.

[3] Erwei Wang.A study of face detection and recognition on Android platform[D]. XiDian university, 2013.

[4] Wei Lv.Research on face detection and recognition based on Android[D].Southwest Jiaotong University, 2014.

[5] Lu, K. and L. Dong. Using LBP histogram for face recognition on Android platform. in International Conference on Computer Research and Development.Shanghai(CN).

[6] Weiguo Yang, Shuohe Zheng, Fast Smile Recognition Method for Mobile Platform. Journal of System Simulation, 2012. 24(1):p. 86-89.

[7] Xiaoping Hu, Duansheng Chen, Chen Weibin. Design and implementation of face detection system on smart phone [J]. Computer Engineering and Design, 2010 (03):672-675.

[8] Yuelong Li, Yan Jin, Wang Jianming, et al. Face feature points extraction:a review[J]. Chinese Journal of Computers 2016,39(7):1356-1374.

[10] Bingbing Zhang. Research on Face Recognition under Changing Light Conditions and Implementation on Android [D]. XiDian university, 2014. 